Auch im Pflegebereich geht nichts mehr ohne „Kollege“ Computer. Wir stellen Ihnen hier nicht nur die wichtigsten Software-Anwendungen für die Pflege vor, sondern beantworten auch Fragen

\title{
Patientendatenmanagementsysteme (PDMS)
}

\section{Sicherheit auf der ITS}

\author{
Auf Intensivstationen sind heute eine Vielzahl an Hightech-Medizin- \\ geräten im Einsatz, die detaillierte Daten zum Patienten liefern. \\ Auch die Teams auf einer ITS erheben deutlich mehr Informationen \\ als auf peripheren Stationen. Doch wie wird man der Datenmengen \\ Herr? Unterstützung leisten hier immer häufiger so genannte \\ Patientendatenmanagementsysteme (PDMS).
}

zu Datensicherheit und Datenschutz.

1.Pflegeinformationssysteme

2. Dienstplanprogramme

3. Patientendatenmanagement

4. Material- und Apothekenbestellung

5. Datensicherheit und Datenschutz

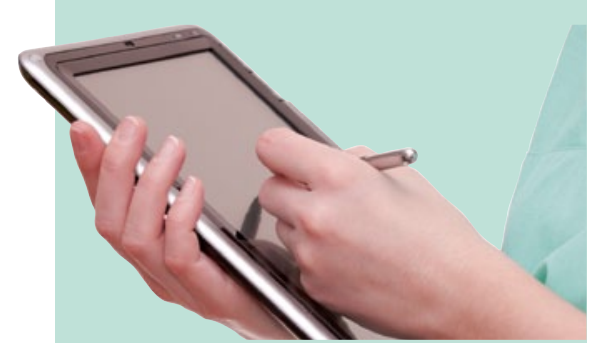

ine Hauptanforderung an speziell auf die Intensivmedizin zugeschnittene Softwarelösungen ist die weitgehend papierlose Dokumentation von Patienteninformationen, Vital- und Gerätedaten sowie Labor- und Therapieinformationen. Dafür werden möglichst alle am Patienten zum Einsatz gebrachten Medizingeräte wie Vitalmonitoring, Beatmungs- oder Dialysegeräte an das PDMS angebunden und die erhobenen Daten lückenlos dokumentiert. Das vermeidet Übertragungsfehler und reduziert den Arbeitsaufwand der Pflegefachkräfte.

\section{Papierlos und sicher}

Die medizinische und pflegerische Dokumentation erfolgt in speziell angepasste und standardisierte Programmformulare. Häufig sind hier bereits Leitlinien, Standards und Fachinformationen hinterlegt. Das ermöglicht eine schnelle Erfassung mittels Auswahlfeldern und Checkboxen und unterstützt aktiv den Workflow. Die Daten werden automatisch auf Plausibilität (z.B. Medikamenteninteraktionen, Fehldosierungen) geprüft. Auch die häufigsten Scores (z.B. TISS, SAPS, IMKB), werden von den meisten PDMS überwie- gend automatisch generiert, was die Dokumentationsqualität und Informationsdichte erheblich erhöht.

Auch für das Medizincontrolling ist das PDMS eine wertvolle Unterstützung. Kodierfachkräfte müssen nicht mehr große Mengen unterschiedlichster Intensivkurven nach abrechnungsrelevanten Informationen durchsuchen. Diese werden automatisch an die Abrechnungssoftware übertragen. Beatmungszeiten oder abrechenbare Medikamente gehen nicht mehr verloren. Auch die Doppeldokumentation von Leistungsdaten wird vermieden. Mit der hohen Qualität der Dokumentation erfüllen die Einrichtungen auch ihre gesetzlichen Dokumentationspflichten wie Vollständigkeit, Reproduzierbarkeit, Verfügbarkeit und Nachvollziehbarkeit der Patientenversorgung.

\section{Software als Medizinprodukt}

Moderne PDMS können aber noch mehr: Zunehmend werden sie zum Management von Infusions- und Spritzenpumpen eingesetzt. Das System erkennt automatisch den Betrieb und Veränderungen (z.B. Bolusgabe) an den Pumpen, die applizierten Medikamente und dokumentiert bezie- hungsweise bilanziert die Therapie. Auch das frühzeitige Erkennen einer beginnenden Sepsis ist mit speziellen Funktionen (Watchdog) im PDMS möglich.

Schon heute stellt ein PDMS die Daten an jedem Ort bereit, an dem sie benötigt werden. Dies kann sowohl bettseitig als auch dezentral in der Klinik sein. Viele Hersteller stellen auch Apps für mobile Geräte und Tablett-PCs zur Verfügung.

Durch die steigende Komplexität der Software und ihren Einsatz in einem sehr sensiblen Bereich sind die Anforderungen an Qualität, Stabilität und Verfügbarkeit eines PDMS sehr hoch. Auch deshalb nehmen die Hersteller ihre Verantwortung sehr ernst und vertreiben die Software zunehmend als Medizinprodukt. Damit ergeben sich für den Betreiber die gleichen Anforderungen (z.B. Einweisungen) wie für andere Medizinprodukte. Dies soll die Risiken beim Einsatz eines PDMS reduzieren und die Patientensicherheit erhöhen. Schließlich werden in dem System eine Fülle von Daten zur akuten Diagnostik oder Therapie genutzt.

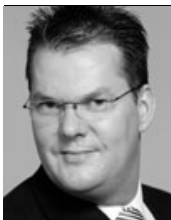

Heiko Mania, M.Sc., MBA Nordstr. 53, 50170 Kerpen mania@online.de h.mania@sana-mtsz.de www.gesundheits-it.com Der Autor ist ausgebildeter Krankenpfleger, Gesundheitsinformatiker und IT-Projektmanager. Bei der Sana-MTSZ GmbH ist er im Bereich „Medizintechnik \& IT (MIT)“ tätig. 